\title{
Isolation and Antimicrobial Sensitivity Patterns of Enteric Bacterial Pathogens from Asymptomatic Food Handlers, Jimma, Ethiopia
}

\author{
Teshale Worku ${ }^{1}$, Ayalew Jejaw ${ }^{1,}$, , Subbaram Kannan², Beyene Wondafrash ${ }^{2}$ \\ ${ }^{1}$ Department of Biomedical Science, College of Health Sciences, Mizan Tepi University, MizanTeferi, Ethiopia \\ ${ }^{2}$ Departmentof Medical Laboratory Sciences, College of Health and Medical Sciences, Jimma University, Jimma, Ethiopia
}

\section{Email address:}

teshaleworku@gmail.com (T. Worku), ayalewjejaw@yahoo.com (A. Jejaw), beyenewondafrash@googlemail.com (B. Wondafrash), subbaramkannan@gmail.com (S. Kannan)

\section{To cite this article:}

Teshale Worku, Ayalew Jejaw, Subbaram Kannan, Beyene Wondafrash. Isolation and Antimicrobial Sensitivity Patterns of Enteric Bacterial Pathogens from Asymptomatic Food Handlers, Jimma, Ethiopia. American Journal of Health Research. Vol. 3, No. 6, 2015 , pp. $399-406$. doi: 10.11648/j.ajhr.20150306.24

\begin{abstract}
Background: Most enteric bacteria are harmless but species which belong to Salmonella, Shigella, Yersinia and certain strains of Escherichia coli are pathogens. The emergences of increased antimicrobial resistances are global challenges, particularly in developing countries due to misuse of antimicrobial agents. This study was aimed at determining the prevalence and antimicrobial sensitivity patterns of enteric bacterial pathogens isolated from food handlers in Jimma Town, Ethiopia. Methods: A cross-sectional study was conducted from July 16/2012 to November 23/2012 among 218 food handlers. Structured questionnaire was used to assess associated factors for enteric bacterial infection. Stool samples were collected andantimicrobial sensitivity tests were done using Mueller Hinton Agar (MHA) by Kirby-Bauer disc diffusion method following standard procedures. Descriptive statistics were performed using SPSS version 16.P-values less than 0.05 were considered as statistically significant. Results: The prevalence of enteric bacterial pathogens was found to be $6.9 \%(15 / 218)$. All $S$. typhi isolates showed resistance to two antimicrobials; amoxicillin-clavulanic acid and streptomycin. However, they all showed sensitivity to most antimicrobials. Half of Shigella isolates showed resistance to two antimicrobials; chloramphenicol and trimethoprim-sulfamethoxazole where as the other half showed resistant toother four antimicrobials; amoxicillin-clavulanic acid, ampicillin, streptomycin and tetracycline. One-third of enterohaemorrhagic Escherichia coli (EHEC) O157:H7 strains of the isolates were found to be resistant to two antimicrobials; amoxicillin-clavulanic acid and tetracycline. Enteric bacterial infection had a significant association with poor training in food handling and preparation $(\mathrm{p}=0.034)$, not knowledgeable about food borne infection $(\mathrm{p}<0.001)$ and inconsistent hand washing $(\mathrm{p}=0.033)$. Conclusion: Most of the isolates were resistant to ampicillin, streptomycin and tetracycline while all were sensitive to ciprofloxacin and nalidixic acid. Health education along with continuous food safety training should be given to food handlers so that they can adhere with effective hygienic practices.
\end{abstract}

Keywords: Antimicrobial, Enteric Bacterial Pathogens, Food Handler, Sensitivity

\section{Background}

Enteric bacteria are microbes that reside in the guts of animals and humans. The human gut is therefore the natural habitat for various bacteria species and majority of them participate in metabolic activities that salvage energy and absorbable nutrients protecting the colonized host against invasion by alien microbes and important atrophic effects on intestinal epithelia and on immune structure and function. Thus, they play an essential role in the development and homeostasis of the immune system [1].

Enteric bacterial pathogens, the major causes of food borne gastroenteritis in humans, remain important health problems worldwide. Such infections associated with food contamination are the major public health problems especially in developing countries resulting in morbidity, mortality and socioeconomic impacts such as high rates of hospitalizations and high treatment costs [2]. Currently, one of the major reasons for morbidity as well as mortality associated with gastrointestinal infections is the increasing resistance of the organisms for available antimicrobial agent 


\section{[2], [3]-[4].}

Food handlers who harbor enteric bacteria asymptomatically and who are not adhered with good hygienic practices and inadequate knowledge on food safety are likely to contaminate the food with enteric bacterial pathogens and could also be the source of food borne infections [4]. However, the consequences of food contamination vary among countries and regions of the world depending on climate, geography and degree of social and economic development [4], [5], [6] - [7].

Since food handlers are engaged in food preparation, transport and provision, they are implicated for the transmission of enteric bacterial pathogens to the community if appropriate hygienic practices are not habituated [7], [8] - [9].

Indeed a prerequisite for the prevention and control of diseases due to enteric bacterial pathogens is a clear understanding of their epidemiology. Therefore, an effective means of prevention of their transmission from food handler to consumers is strict adherence to good personal hygiene and hygienic food handling practices.However, for instance, hand washing, being a simple and effective way to cut down cross-contamination, yet often forgotten [5], [10].

People commonly experience dinning out in food establishments such as Hotels, Restaurants and Cafeterias. These establishments prepare and provide various food items to many people implying a possible risk of infection if sanitary and hygienic conditions are not strictly followed [7], [9]. Although certain studies were conducted in some parts of Ethiopia, holistic information regarding enteric bacterial infections and risk factors among food handlers in many parts of the country including the study area is limited. Moreover, emergence or rise in drug resistant enteric bacterial pathogens is a major public health concern needed to be addressed recently. Hence, this study is aimed at determining the prevalence and antimicrobial sensitivity patterns of enteric bacterial pathogens isolated from food handlers found in Jimma town, southwest Ethiopia.

\section{Methods}

\subsection{Study Area}

The study was conducted in Jimma town which is located $350 \mathrm{kms}$ south west of Addis Ababa (the capital city of Ethiopia). It is located at an average altitude of 1780 meters above sea level with geographical coordinates of approximately $7^{\circ} 41^{\prime} \mathrm{N}$ latitude and $36^{\circ} 50^{\prime} \mathrm{E}$ longitude. It lies in the climatic zone locally known as 'Woyna Dega' (areas located between $1,500-2,400 \mathrm{~m}$ above sea level) which is considered ideal for agriculture as well as human settlement. The town is generally characterized by warm climate with mean annual maximum temperature of $30^{\circ} \mathrm{C}$ and mean annual minimum temperature of $14^{\circ} \mathrm{C}$. The annual rainfall ranges from 1138 to $1690 \mathrm{~mm}$. According to the central statistical agency (CSA) of 2007, the total population of the town is 130,254. The town has various food establishments such as Hotels, Restaurants and Cafeterias which serve for the people of the town as well as the visitors.

\subsection{Study Design and Sample Size Determination}

Cross-sectional study was conducted from July 16/2012 to November 23/2012 among food handlers working at food establishments found in Jimma town. Sample size was determined using single population proportion formula $n=Z^{2}$ $\mathrm{p}(1-\mathrm{p}) / \mathrm{d}^{2}$, with the following assumptions: prevalence (p) of $17 \%$ from a previous study in Nigeria [11], 95\% confidence level, 5\% margin of error, and $10 \%$ for anticipated non-response rate. Accordingly, the minimum sample size (n) was found to be 239 . The total sample size was allocated proportionally to 102 Hotels, 94 Restaurants and 86 Cafeterias. Accordingly, 86, 80 and 73 participants were planned to be included from each selected food establishment in the study. But, 78, 72, and 68 participants were included giving a total of 218 participants (91.2\% response rate). Lottery method random sampling procedure was used to recruit study participants.

\subsection{Data Collection}

\subsubsection{Demographic and Personal Risk Factors Survey}

Data on demographic profile of the participants' and factors predisposing to enteric bacterial pathogens was gathered using pretested questionnaire. The questions included information on sex, age, educational status, and hand washing practice are among the others.

\subsubsection{Stool Sample Collection}

Stool samples were collected using clean, dry and leak proof stool cups. The specimens were then immediately placed in to Cary-Blair transport medium. The samples were then transported to Jimma University Medical Microbiology Laboratory in ice packed box for bacterial culture and identification.

\subsection{Sample Processing and Identification of Bacteria}

For isolation of enteric bacteria, stool samples were directly inoculated onto MacConkey agar. The samples were also sub-cultured on to Xylose Lysine Deoxycholate (XLD) agar after enrichment with Selenite cystine broth (prepared in $10 \mathrm{ml}$ amounts) and incubated at $37^{\circ} \mathrm{C}$ for $18-24$ hours. After 24 hours of incubation at $37^{\circ} \mathrm{C}$, isolates were identified following the standard procedures using biochemical tests hydrogen sulfide $\left(\mathrm{H}_{2} \mathrm{~S}\right)$ production, indole production and motility in Sulfide-Indole-Motility (SIM) medium, citrate utilization, urease production, different carbohydrate fermentation reactions and Lysine decarboxylase (LDC) in Simmon's Citrate agar, Urea agar, Kligler's iron agar (KIA) and lysine iron agar (LIA) [12]. All the biochemical media wereobtained from Oxoid, (Hampshire, England) (Fig 1).

\subsection{Antimicrobial Susceptibility Testing}

Antimicrobial sensitivity test procedures and they were done using Mueller Hinton Agar (MHA) by Kirby-Bauer disc diffusion method following standard procedures (Fig 2). The following antimicrobial agents were used for the isolates: amoxicillin-clavulanic acid (AML, 30 $\mathrm{g}$ ), ampicillin (AMP, 
$10 \mu \mathrm{g})$, chloramphenicol (C, 30 $\mu \mathrm{g})$, ciprofloxacin (CIP, $5 \mu \mathrm{g})$, gentamicin $(\mathrm{CN}, 10 \mu \mathrm{g})$, ceftriaxone $(\mathrm{CRO}, 30 \mu \mathrm{g})$, nalidixic

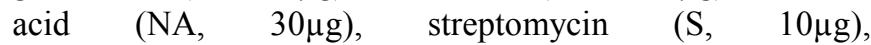
trimethoprim-sulfamethoxazole (TS, $25 \mu \mathrm{g})$ and tetracycline

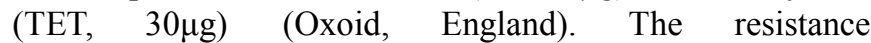
andsensitivitywere interpreted according to the Standards.[13]

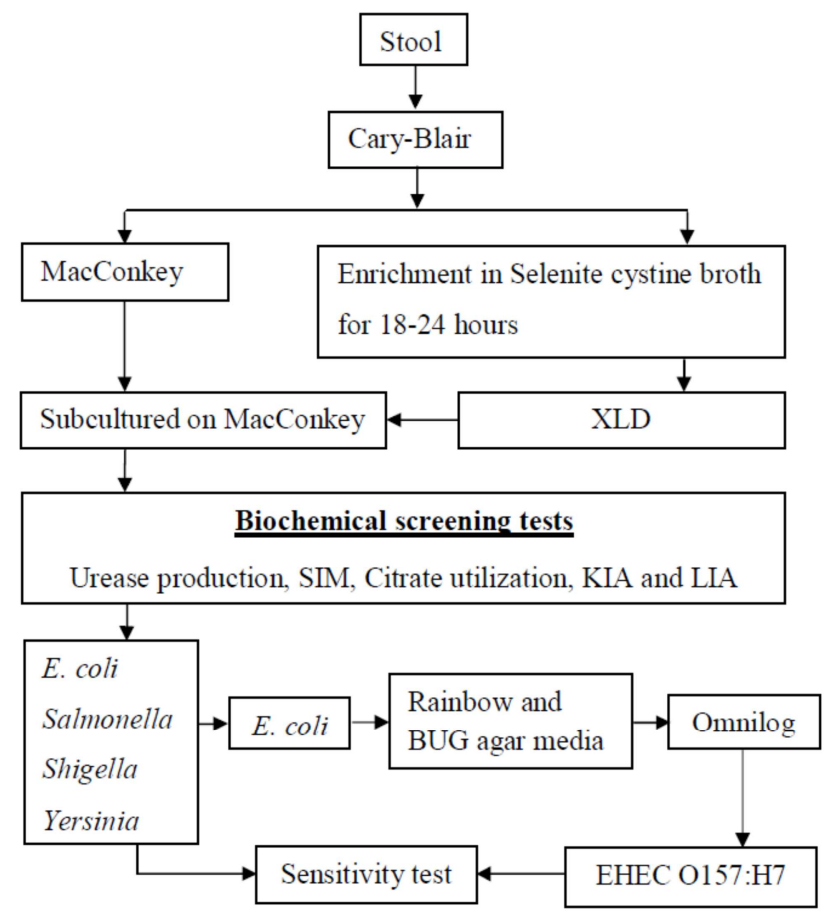

Figure 1. Flow diagram showing culture and identification procedures followed for enteric bacterial pathogens isolated from food handlers, Jimma, Southwest Ethiopia, 2012.

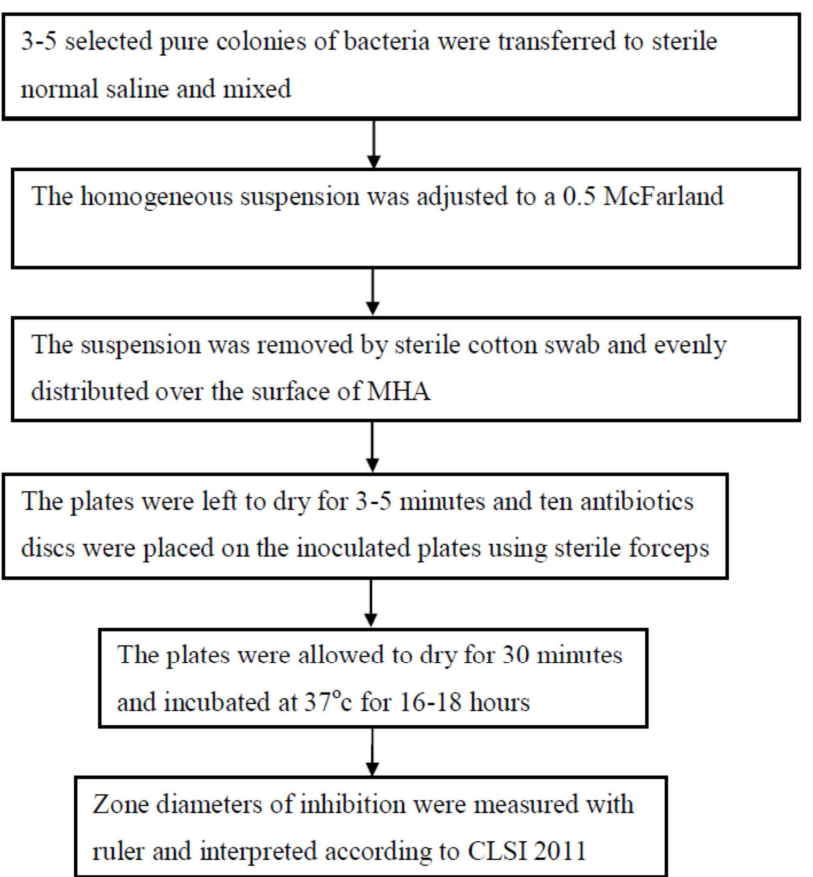

Figure 2. Antimicrobial sensitivity test procedures followed for enteric bacterial pathogens isolated from food handlers, Jimma, Southwest Ethiopia, 2012.

\subsection{Data Analysis}

Data were coded, checked for completeness and cleaned for any inconsistencies. The data were then entered and analyzed using SPSS version 16.0. Descriptive statistics were used and $5 \%$ level of precision was used for checking the association between dependent and independent variables.

\subsection{Ethical Clearance}

Approval was secured from the Ethical Clearance Committee of Jimma University. Formal letter was also written from the Municipality of the town to food establishments and informed consent was obtained from each study participant. Individuals who were found positive for the detected organisms were treated by the effective antibiotics.

\section{Results}

\subsection{Socio-demographic Characteristics}

A total of 218 food handlers were participated in this study and their socio-demographic characteristics were presented below (Table1).

Table 1. Socio-demographic characteristics of selected food handlers, Jimma, Southwest Ethiopia, 2012.

\begin{tabular}{llll}
\hline Characteristics & & Frequency & Percent (\%) \\
\hline Sex & Male & 63 & \\
& Female & 155 & 28.9 \\
Age category (Years) & & & 71.1 \\
& $15-24$ & 71 & 32.6 \\
& $25-34$ & 114 & 52.3 \\
& $35-44$ & 23 & 10.6 \\
Educational Status & $45-52$ & 10 & 4.6 \\
& & & \\
& Illiterate & 29 & 13.3 \\
& Read and write & 22 & 10.1 \\
& Literate & 167 & 76.6 \\
& $1-8$ & 84 & 38.5 \\
& $9-12$ & 63 & 28.9 \\
& Above 12 & 20 & 9.2 \\
\hline
\end{tabular}

\subsection{Isolation Rates of Enteric Bacterial Pathogens}

As presented on figure3, the status of infection with enteric bacterial pathogens among the subjects in this study consists of the following types of isolates; $1.8 \%(4 / 218)$ EHEC O157:H7, 2.7\% (6/218) Salmonella species, 1.4\% (3/218) S. typhi, and $0.9 \%$ (2/218) Shigella species giving a total of $6.9 \%$ $(15 / 218)$ isolates. Therefore, the prevalence of enteric bacterial pathogens among food handlers participated in this study was $6.9 \%$. Multiple infections were not detected in any of the processed samples. In describing the prominent isolates of the total enteric bacterial pathogens detected, the dominant species were found to be untyped Salmonella species consisting of $40 \%(6 / 15)$ followed by EHEC O157:H7 which accounts $26.7 \%(4 / 15)$, S. typhi $20 \%(3 / 15)$ and Shigella species $13.3 \%(2 / 15)$. 


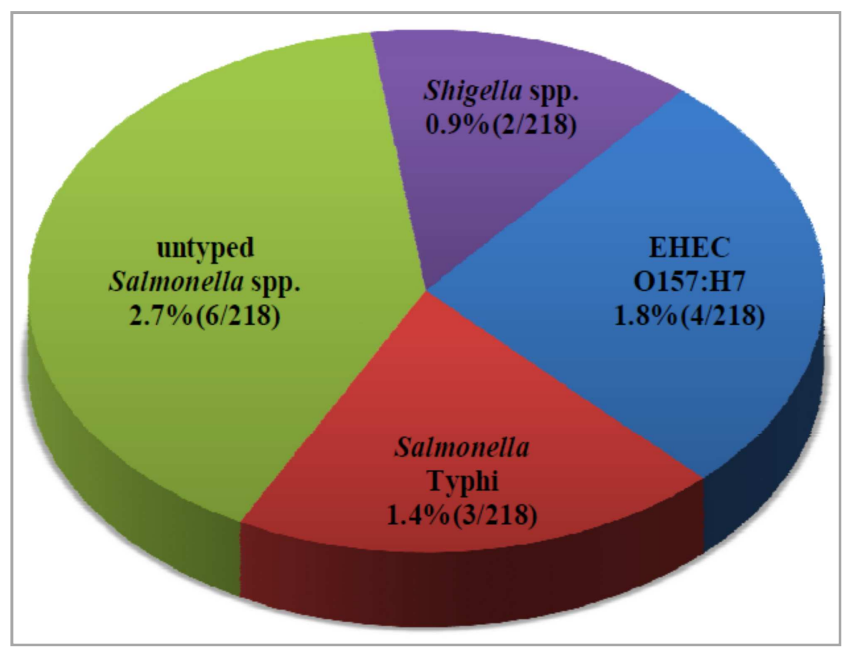

Figure 3. Species and strains of enteric bacterial pathogens isolated from food handlers, Jimma, Southwest Ethiopia, 2012.

\subsection{Antimicrobial Sensitivity Patterns of Enteric Bacterial Pathogens}

Ten antimicrobial discs were used for sensitivity tests of the isolated enteric bacterial pathogens following standard procedures. Accordingly, the isolates showed varied sensitivities to the tested antimicrobials and the results were interpreted and presented in tables for each of them as; resistant (R), intermediate (I) and sensitive (S) according to the CLSI 2011 guideline.

\subsubsection{Antimicrobial Sensitivity Patterns of S. typhi and Untyped Salmonella Species}

As presented on Table 2 all the three (100\%) S. typhi isolates showed resistance to two antimicrobials; amoxicillin-clavulanic acid and streptomycin. But, only one (33.3\%) isolate showed resistance to ampicillin.However, all $(100 \%)$ of the isolates showed sensitivity to the rest antimicrobials; ceftriaxone, chloramphenicol, ciprofloxacin, gentamicin, trimethoprim-sulfamethoxazole and nalidixic acid. Unlike S. typhi, 83.3\% (5/6) Salmonella species were resistant to amoxicillin-clavulanic acid followed by $66.7 \%$ $(4 / 6)$ of the isolates which showed resistance to ampicillin. Referring the above species, 50\% (3/6) of the isolates showed resistance to streptomycin and tetracycline and 33.3\% (2/6) were resistant to trimethoprim-sulfamethoxazole. No resistance was observed by any of the Salmonella species as well as $S$. typhi isolates to ciprofloxacin and nalidixic acid.

Table 2. Antimicrobial sensitivity patterns of S. typhi and untyped Salmonella species isolates from selected food handlers, Jimma, Southwest Ethiopia, 2012.

\begin{tabular}{|c|c|c|c|c|c|c|}
\hline \multirow{2}{*}{ Amc } & \multicolumn{3}{|c|}{ Salmonella typhi } & \multicolumn{3}{|c|}{ Untyped Salmonella species } \\
\hline & $\mathbf{R} \%$ & $\mathbf{I} \%$ & $\mathbf{S} \%$ & $\mathbf{R} \%$ & I\% & $\mathbf{S} \%$ \\
\hline AML & $100(3 / 3)$ & $0(0 / 3)$ & $0(0 / 3)$ & $83.3(5 / 6)$ & $0(0 / 6)$ & $16.7(1 / 6)$ \\
\hline AMP & $33.3(1 / 3)$ & $0(0 / 3)$ & $66.7(2 / 3)$ & $66.7(4 / 6)$ & $0(0 / 6)$ & $33.3(2 / 6)$ \\
\hline CRO & $0(0 / 3)$ & $0(0 / 3)$ & $100(3 / 3)$ & $16.7(1 / 6)$ & $0(0 / 6)$ & $83.3(5 / 6)$ \\
\hline $\mathrm{C}$ & $0(0 / 3)$ & $0(0 / 3)$ & $100(3 / 3)$ & $16.7(1 / 6)$ & $16.7(1 / 6)$ & $66.6(4 / 6)$ \\
\hline CIP & $0(0 / 3)$ & $0(0 / 3)$ & $100(3 / 3)$ & $0(0 / 6)$ & $0(0 / 6)$ & $100(6 / 6)$ \\
\hline $\mathrm{CN}$ & $0(0 / 3)$ & $0(0 / 3)$ & $100(3 / 3)$ & $16.7(1 / 6)$ & $0(0 / 6)$ & $83.3(5 / 6)$ \\
\hline NA & $0(0 / 3)$ & $0(0 / 3)$ & $100(3 / 3)$ & $0(0 / 6)$ & $0(0 / 6)$ & $100(6 / 6)$ \\
\hline $\mathrm{S}$ & $100(3 / 3)$ & $0(0 / 3)$ & $0(0 / 3)$ & $50(3 / 6)$ & $0(0 / 6)$ & $50(3 / 6)$ \\
\hline TET & $0(0 / 3)$ & $66.7(2 / 3)$ & $33.3(1 / 3)$ & $50(3 / 6)$ & $16.7(1 / 6)$ & $33.3(2 / 6)$ \\
\hline
\end{tabular}

Key: Amc $=$ Antimicrobial, $\mathrm{AML}=$ Amoxicillin-Clavulanic acid, $\mathrm{AMP}=$ Ampicillin, $\mathrm{CRO}=\mathrm{Ceftriaxone}, \mathrm{C}=\mathrm{Chloramphenicol}, \mathrm{CIP}=\mathrm{Ciprofloxacin}, \mathrm{TS}=$ Trimethoprim-Sulfamethoxazole, $\mathrm{CN}=$ Gentamicin, $\mathrm{NA}=$ Nalidixic Acid, $\mathrm{S}=$ Streptomycin, $\mathrm{TET}=$ Tetracycline, $\mathrm{R}=\mathrm{Resistant}, \mathrm{I}=\mathrm{Intermediate}, \mathrm{S}=\mathrm{Sensitive}$, and $\%=$ Percent

Table 3. Antimicrobial sensitivity patterns of EHEC O157:H7 and Shigella species isolates from selected food handlers, Jimma, Southwest Ethiopia, 2012.

\begin{tabular}{|c|c|c|c|c|c|c|}
\hline \multirow{2}{*}{ Amc } & \multicolumn{3}{|c|}{ Shigella species } & \multicolumn{3}{|c|}{ EHEC 0157:H7 } \\
\hline & $\mathbf{R} \%$ & I\% & $\mathbf{S \%}$ & $\mathbf{R} \%$ & I\% & $\mathbf{S \%}$ \\
\hline AML & $50(1 / 2)$ & $0(0 / 2)$ & $50(1 / 2)$ & $75(3 / 4)$ & $0(0 / 4)$ & $25(1 / 4)$ \\
\hline AMP & $50(1 / 2)$ & $0(0 / 2)$ & $50(1 / 2)$ & $50(2 / 4)$ & $0(0 / 4)$ & $50(2 / 4)$ \\
\hline $\mathrm{CRO}$ & $0(0 / 2)$ & $0(0 / 2)$ & $100(2 / 2)$ & $25(1 / 4)$ & $25(1 / 4)$ & $50(2 / 4)$ \\
\hline $\mathrm{C}$ & $50(1 / 2)$ & $0(0 / 2)$ & $50(1 / 2)$ & $25(1 / 4)$ & $25(1 / 4)$ & $50(2 / 4)$ \\
\hline CIP & $0(0 / 2)$ & $0(0 / 2)$ & $100(2 / 2)$ & $0(0 / 4)$ & $0(0 / 4)$ & $100(4 / 4)$ \\
\hline $\mathrm{CN}$ & $0(0 / 2)$ & $0(0 / 2)$ & $100(2 / 2)$ & $25(1 / 4)$ & $0(0 / 4)$ & $75(3 / 4)$ \\
\hline NA & $0(0 / 2)$ & $0(0 / 2)$ & $100(2 / 2)$ & $0(0 / 4)$ & $0(0 / 4)$ & $100(4 / 4)$ \\
\hline S & $50(1 / 2)$ & $0(0 / 2)$ & $50(1 / 2)$ & $25(1 / 4)$ & $0(0 / 4)$ & $75(3 / 4)$ \\
\hline TET & $50(1 / 2)$ & $0(0 / 2)$ & $50(1 / 2)$ & $75(3 / 4)$ & $0(0 / 4)$ & $25(1 / 4)$ \\
\hline
\end{tabular}

Key: Amc $=$ Antimicrobial, $\mathrm{AML}=$ Amoxycillin-Clavulanic acid, $\mathrm{AMP}=$ Ampicillin $, \mathrm{CRO}=\mathrm{Ceftriaxone}, \mathrm{C}=\mathrm{Chloramphenicol}, \mathrm{CIP}=\mathrm{Ciprofloxacin}, \mathrm{EHEC}=$ Enterohaemorrhagic E. coli, TS = Trimethoprim-Sulfamethoxazole, $\mathrm{CN}=$ Gentamicin, $\mathrm{NA}=$ Nalidixic Acid, $\mathrm{S}=\mathrm{Streptomycin}$, TET $=$ Tetracycline, $\mathrm{R}=$ Resistant, I = Intermediate, $\mathrm{S}=$ Sensitive, and $\%=$ Percent 


\subsubsection{Antimicrobial Sensitivity Patterns of Shigella Species and $\mathrm{EHEC} \mathrm{O157:H7}$}

As presented on the table 3 half (50\%) of Shigella isolates showed resistance to six antimicrobials; Amoxycillin-Clavulanic acid, Ampicillin, Chloramphenicol, Trimethoprim-Sulfamethoxazole, Streptomycin, and Tetracycline. However, $100 \%$ of the isolates were found to be sensitive to the remaining four antimicrobials; Ceftriaxone, Gentamicin, Ciprofloxacin, and Nalidixic Acid.

Regarding enterohaemorrhagic Escherichia coli (EHEC) O157:H7 strains, three (75\%) of the isolates were resistant to two antimicrobials; amoxicillin-clavulanic acid and tetracycline. Two $(50 \%)$ of the strains showed sensitivity to four antimicrobials; ampicillin, ceftriaxone, chloramphenicol and trimethoprim-sulfamethoxazole. Three $(75 \%)$ of the strains showed sensitivity to gentamicin and streptomycin. But, all the four $(100 \%)$ strains were sensitive to two antimicrobials; ciprofloxacin and nalidixic acid.

\subsubsection{Multidrug Resistance Pattern of Enteric Bacterial Pathogens}

Two (50\%) of the EHEC O157:H7 strains were multidrug resistant each of them showing resistance to four and eight antimicrobials. Regarding $S$. typhi, one $(33.3 \%)$ of the isolates showed multidrug resistance showing resistant to three of the antimicrobials. However, five $(83.3 \%)$ untyped Salmonella isolates showed multidrug resistance of which two (33.3\%) showed resistance to four antimicrobials; and the remainder showed resistance to three $(16.7 \%)$, five $(16.7 \%)$ and eight (16.7\%) antimicrobials. One (50\%) Shigella showed multidrug resistance to four antimicrobials as it is summarized on table 4.

\subsection{Associated Factors for Enteric Bacterial Infection Among Food Handlers}

Based on chi-square statistical test there was a significant association between enteric bacterial infection and not getting training in food handling and preparation $(\mathrm{p}=0.034)$, not knowledgeable about food borne infection $(\mathrm{p}<0.001)$ and inconsistent hand washing practice $(\mathrm{p}=0.033)$ (Table 5).

Table 4. Multidrug resistance of enteric bacterial pathogens isolates from selected food handlers, Jimma, Southwest Ethiopia, 2012

\begin{tabular}{|c|c|c|c|c|c|}
\hline \multirow{2}{*}{ Organism } & \multicolumn{4}{|c|}{ Resistance (\%) } & \multirow{2}{*}{ Antimicrobial agents } \\
\hline & $\mathbf{R}_{\mathbf{3}}$ & $\mathbf{R}_{4}$ & $\mathbf{R}_{\mathbf{5}}$ & $\mathbf{R}_{8}$ & \\
\hline \multirow{2}{*}{ EHEC O157:H7 } & \multirow{7}{*}{$\begin{array}{l}33.3(1 / 3) \\
16.7(1 / 6)\end{array}$} & $25(1 / 4)$ & \multirow{7}{*}{$16.7(1 / 6)$} & \multirow{3}{*}{$25(1 / 4)$} & AML, AMP, TS and TET \\
\hline & & & & & AML, AMP, CRO, C, TS, CN, S and TET \\
\hline S. typhi & & & & & AML, AMP and S \\
\hline \multirow{3}{*}{ Untyped Salmonella species } & & & & & $\mathrm{C}, \mathrm{S}$ and TET \\
\hline & & $33.3(2 / 6)$ & & & AML, AMP, TS and TET \\
\hline & & & & & AML, AMP, C, S and TET \\
\hline Shigella species & & $50(1 / 2)$ & & & AML, AMP, S and TET \\
\hline
\end{tabular}

Key: $\mathrm{AML}=$ Amoxicillin-Clavulanic acid, $\mathrm{AMP}=$ Ampicillin, $\mathrm{CRO}=$ Ceftriaxone, $\mathrm{C}=$ Chloramphenicol, EHEC $=$ Enterohaemorrhagic Escherichia coli, TS $=$ Trimethoprim-Sulfamethoxazole, $\mathrm{CN}=$ Gentamicin, $\mathrm{S}=$ Streptomycin, $\mathrm{TET}=$ Tetracycline, $\mathrm{R}_{3}=$ resistant to three antimicrobials, $\mathrm{R}_{4}=$ resistant to four antimicrobials, $R_{5}=$ resistant to five antimicrobials, $R_{8}=$ resistant to eight antimicrobials and $\%=$ Percent

Table 5. Associated factors Enteric Bacterial Pathogens from asymptomatic Food Handlers, Jimma, Southwest Ethiopia, 2012.

\begin{tabular}{|c|c|c|c|c|c|}
\hline \multirow{2}{*}{ Variables } & & \multicolumn{2}{|c|}{ Entericbacterial infection } & \multirow{2}{*}{ Total } & \multirow{2}{*}{ P-value } \\
\hline & & $+\mathrm{Ve}$ & $-\mathrm{Ve}$ & & \\
\hline \multirow{2}{*}{ Training } & Trained & $6(4.2 \%)$ & $136(95.8 \%)$ & $142(100 \%)$ & $X^{2}=4.48$ \\
\hline & Not trained & $9(11.8 \%)$ & $67(88.2 \%)$ & $76(100 \%)$ & 0.034 \\
\hline \multirow{2}{*}{ Medical check up } & Yes & $5(4.2 \%)$ & $113(95.8 \%)$ & $118(100 \%)$ & $X^{2}=2.8$ \\
\hline & No & $10(10 \%)$ & $90(90 \%)$ & $100(100 \%)$ & 0.094 \\
\hline \multirow{2}{*}{ Knowledge about FBI } & Yes & $9(4.6 \%)$ & $188(95.4 \%)$ & $197(100 \%)$ & $X^{2}=17$ \\
\hline & No & $6(28.6 \%)$ & $15(71.4 \%)$ & $21(100 \%)$ & $<0.001$ \\
\hline $\begin{array}{l}\text { Consistency of hand } \\
\text { washing }\end{array}$ & Inconsistent & $11(10.8 \%)$ & $91(89.2 \%)$ & $102(100 \%)$ & 0.033 \\
\hline
\end{tabular}

Key: FBI $=$ Foodborne infection, $+\mathrm{Ve}=$ Infected, $-\mathrm{Ve}=$ Not infected, $\mathrm{X}^{2}=$ calculated value

\section{Discussion}

During the five month study period, 218 asymptomatic food handlers were enrolled from selected food establishments.A total of $15(6.9 \%)$ enteric bacterial pathogens were isolated and their antimicrobial sensitivity patterns were determined. In addition, associated factors for enteric bacterial infection among food handlers were assed and all the results were compared with the findings of other studies.
In this study $1.4 \%$ Salmonella typhi was isolated from food handlers and this is in agreement with the Study conducted from Bahir Dar [8] in which its isolation rate was found to be $1.6 \%$. In the contrary, higher prevalence of Salmonella typhi from India [14] and Nigeria [11] was isolated. In the present study, untyped Salmonella species were also isolated and they account $2.7 \%$. Correspondingly, study done in North India found that $2.5 \%$ Salmonella species were recovered from the stools of food handlers. However, incomparable result was 
obtained from Sudan that found 3.8\% Salmonella species [15]. On the other hand, studies done in Gondar and Egypt revealed that no Salmonella species were isolated from the stools of food handlers [16], [17]. The reason for the difference in the existence of Salmonella species as well as other enteric bacterial pathogens in the stools might be due to variation in climate, geography and study settings.

In this study, isolation rate of Shigella species was found to be $0.9 \%$, and it is lower than the results of Gondar $(3.1 \%)$, and India (9.33\%) [7], [16]. Regarding enterohaemorrhagic Escherichia coli (EHEC) O157:H7 1.8\% strains were isolated and this in agreement with the study conducted from Kenya which was $2.1 \%$ [18]. On the other hand, higher isolation rate was reported from Japan (8.25\%) [17].

As far as antimicrobial sensitivity patterns of enteric bacterial pathogens is concerned; generally, the isolates showed varied sensitivities to ten tested antimicrobials. Hence, there was variation in drug sensitivity patterns among $S$. typhi isolates compared to other studies done somewhere else. For instance, study done in Bahir Dar found that only one (16.7\%) $S$. typhi isolate showed resistance to six antimicrobials; ampicillin, trimethoprim-sulfamethoxazole, tetracycline, chloramphenicol, gentamicin and norfloxacin [8]. However, in the current study, $33.3 \% S$. typhi showed resistance to three antimicrobials; amoxicillin-clavulanic acid, ampicillin and streptomycin. On the other hand, study conducted in India reported that $78.4 \% S$. typhi showed resistance to three antimicrobials; ampicillin, chloramphenicol and trimethoprim-sulfamethoxazole which are conventionally used to treat typhoid fever. Least resistance to norfloxacin, ciprofloxacin, gentamicin and nalidixic acid was observed by most isolates, as reported in the above study. However, multidrug resistance in this study showed by $33.3 \%$ of the isolates differs from the above study where $83.3 \%$ of the isolates were found to be multidrug resistant [14]. The variation might be due to sample size difference.

All untyped Salmonella species were sensitive to ciprofloxacin and nalidixic acid where as five (83.3\%) were found to be multidrug resistant. In coherent with the present findings, sensitivity to ciprofloxacin and nalidixic acid and frequent resistance to amoxicillin-clavulanic acid, ampicillin and tetracycline among Salmonella species was also reported from studies conducted somewhere. For instance, study done in Nigeria found that $96 \%$ and $27.6 \%$ Salmonella species were found to be sensitive to ciprofloxacin and chloramphenicol, respectively. However, the isolates showed complete resistance to tetracycline, ampicillin and amoxicillin [11]. According to the study conducted in Sudan, 93.1\% Salmonella species were found to be resistant to the above mentioned antimicrobials and $47.1 \%$ isolates showed multidrug resistance [19].

As the antimicrobial sensitivity test results imply, $100 \%$ of the Shigella isolates were sensitive to two antimicrobials; ciprofloxacin and nalidixic acid. Differently, result reported from China found that $96.4 \%$ Shigella isolates were resistant to nalidixic acid. Multidrug resistance observed on $50 \%$ of the isolates in this study also differs from the report of the same study where $91 \%$ of the isolates showed multidrug resistance [20]. Sensitivity to ciprofloxacin and gentamicin Shigella isolates in the present study agrees with the result found from Gondar University where $91.1 \%$ and $92.1 \%$ were found to be sensitive to ciprofloxacin and gentamicin, respectively. Resistance to at least three antimicrobials reported from the above study also agrees with the present finding where $50 \%$ of the isolates showed resistance to four antimicrobials [21]. Similarly, result reported from a separate study done in Gondar University found all Shigella isolates which were sensitive to ciprofloxacin but most of them found to be resistant to ampicillin [5].

Concerning the sensitivity patterns of EHEC O157:H7 isolates, least sensitivity to amoxicillin-clavulanic acid and tetracycline agrees with the finding reported from Iran where $89.7 \%$ shiga toxin producing E. coli (STEC) and ETEC were resistant to tetracycline [22]. In agreement with the present finding, study done in Nigeria found that 91.4\% EHEC O157:H7 were resistant to tetracycline [23].

As far as associated factors are concerned, the present study not getting training in food handling and preparation was a significant factor for enteric bacterial infection and it was found that only $15.6 \%$ food handlers have formal certificate of training in food handling and preparation. Correspondingly, studies conducted in Bahir Dar and Mekelle University reported that $14 \%$ and $12.3 \%$ of the food handlers have six months formal certificate of training in food handling and preparation respectively [8], [24]. Differently, study conducted in Gondar University found that only 3\% of the food handlers have formal certificate of training in food handling and preparation [5].

Regarding medical checkup, the present study found that more than half of the food handlers (54.1\%) undergo medical checkup. However, only $13.8 \%$ of them undergo regularly. Similarly, study done in Gondar University found that $53.5 \%$ food handlers undergo medical checkup [5].In addition, results obtained from Mekelle University and Mekelle town reported that $36.8 \%$ and $22.7 \%$ respondents undergo medical checkup respectively [24], [25]. On the other hand, study conducted in Bahir Dar town reported that none of the food handlers undergo medical checkup including stool examination previously [8]. The reason for the variation regarding medical checkup in this study and studies done somewhere else might be due to differences in awareness about food born infections among the owner/managers of food establishments.

Most $90.4 \%$ food handlers had knowledge about food borne infection $(\mathrm{FBI})$, and this is comparable with report from Nigeria (90\%) [11]. Although $90.4 \%$ of study participants in the present study were knowledgeable, insufficient knowledge about food born infection among food handlers was also found to be significant associated factor for enteric bacterial pathogens.

Inconsistent hand washing practice was the other significant associated factor. However, $96.8 \%$ of the food handlers participated in this study always washes their hands before handling food.This finding is in agreement with those 
reported from Bahir Dar town (90.6\%) and Gondar University (94\%) ]5], [8]. Differently, this finding is higher than that found from Nigeria where $72.3 \%$ food handlers wash their hands before preparing food [11]. The reason for the difference in hand washing might be due to variation in hygienic practices that people adhere with from country to country as well as from region to region depending on social and economic development.

\section{Limitations of the Study}

This study was limited to certain enteric bacterial pathogens and other medically important enteric organisms such as Campylobacter and Yersinia enterocolitica were left unidentified due to some constraints. Confirmation of some strains of pathogenic Escherchia coli was also left in this study due to scarcity of Omnilog Microplates. Finger nail samples which might indicate the status of contamination with enteric bacterial pathogens were also not considered in this study.

\section{Conclusions}

The $6.9 \%(15 / 218)$ prevalence of enteric bacterial pathogens in this finding emphasizes that food handler harboring enteric bacterial pathogens asymptomatically are the potential sources food borne infections. All the isolates showed sensitivity to only two antimicrobials; ciprofloxacin and nalidixic acid. Moreover, 33.3\%, Salmonella typhi, 83.3\% untyped Salmonella species, 50\% Shigella species and 50\% EHEC O157:H7 strains showed multidrug resistance. The findings point out that the rise in antimicrobial resistance is still an ongoing public problem in treating enteric bacteria associated infections.Thus, health education is essential to create awareness about food borne infection linked with unhygienic food handling and preparation, Moreover, Continuous supervision and follow up should be undertaken.

\section{Authors' Contributions}

TW conceived the study, involved in the study design, data analysis.BW and SK involved in the design, supervised data collection and analysis.AJ drafted and critically reviewed the manuscript. All authors read and approved the final manuscript.

\section{References}

[1] Tortora GJ, Funke BR, Case CL Microbiology an introduction. 2009; 9thedn, Kidersly, India.

[2] Barrabeig I1 RA, Buesa J, Bartolomé R, Pintó R, Prellezo H, Domínguez A. BMC Infect Dis 2010 Sep 15; doi: 10.1186/1471-2334-10-269;10(269):1471-2334-10-269

[3] DemissieT Andualem, Yehuala F Moges, Fetene D Muluye, Gudeta G Ayalew. Prevalence and antimicrobial susceptibility patterns of Shigella and Salmonella Species among patients with diarrhea attending Gondar town health institutions, Northwest Ethiopia. Science Journal of Public Health. 2014; 2(5):469-75.

[4] Aklilu A, Kahse D, Dessalegn M, Tarekegn N, Gebremichael S, Zenebe S, Desta K, Mamuye Y and Mama M. Prevalence of intestinal parasites, salmonella and shigella among apparently health food handlers of Addis Ababa University student's cafeteria, Addis Ababa, Ethiopia. BMC Research Notes 2015;8(17).

[5] Dagnew M, Moges F, and Gizachew M. Bacterial Profile and Antimicrobial Susceptibility Pattern among Food Handlers at Gondar University Cafeteria, Northwest Ethiopia. J Infect Dis Ther, 2013;1(2):1-6.

[6] Jeo O. Food Safety And Quality Management In Kenya: An Overview Of The Roles Played By Various Stakeholders. African Journal of Food, Agriculture, Nutrition and Development 10(11):4379-97.

[7] Khurana S, Thapar R,Sharma M and, Malla N. Intestinal bacterial and parasitic infections among food handlers in a tertiary care hospital of North India. Tropical Gastroenterology 2008;29(4): 207-9.

[8] Abera B, Biadegelgen F, Bezabih B Prevalence of Salmonella typhi and intestinal parasites among food handlers in Bahir Dar Town, Northwest Ethiopia. Ethiop J Health Dev 2010; 24(1):46-50.

[9] Oladipo I.Incidence of antibiotic resistance in some bacterial pathogens from Street Vended food in Ogbomoso, Nigeria. Pak J Nutr. 2010; 9 (11):1061-8.

[10] Stella I. Smith, Chimere O. Agomo, Moses Bamidele, Bolanle O. Opere, Olusimbo O. Aboaba. Survey of food handlers in bukas (a type of local restaurant) in Lagos, Nigeria about typhoid fever. / HEALTH. 2010;2(8): 951-6.

[11] Stella I. Smith.Antimicrobial susceptibilities of Salmonella isolated from food handlers and cattle in Lagos, Nigeria. International Journal of Health Research. 2009; 2(2):189-93.

[12] Vandepitte J VJ. Basic laboratory Procedures in Clinical Bacteriology. Geneva, Switzerland: World Health Organization; 2003.

[13] National Committee for Clinical Laboratory Standards Methods for determining bactericidal activity of antimicrobial agents. Tentative Guidelines; 2011, M100-TNCCLS. Villanova.

[14] Senthilkumar B, Prabakaran G. Multidrug resistant Salmonella typhi in asymptomatic typhoid carriers among food handlers in Namakkal district Tamil Nadu,. Indian journal of Medical Microbiology. 2005;23 (2):92-4.

[15] Ahmed S.Bacteriological and Parasitological Assessment of Food Handlers in the Omdurman Area of Sudan. J Microbiol Immunol Infect. 2010; 43 (1):70-3.

[16] Andargie G. Kassu A, Moges F, Tiruneh M, Huruy K. Prevalence of Bacteria and Intestinal Parasites among Food handlers in Gondar Town, Northwest Ethiopia. Ethiop J Health Dev 2008; 26(4):451-5.

[17] Bassyouni H. Hefzy H.,Wegdan A. Perception of Food Safety and Prevalence of Staphylococcus aureus and Salmonella species Carriers among Fayoum University Food handlers. Life Science Journal 2012; 9(4):1-7. 
[18] Oundo J., Boga H., Muli F., and Iijima Y. High incidence of enteroaggregative escherichia coli among food handlers in three areas of Kenya: a possible transmission route of travelers' diarrhea. Journal of Travel Medicine. 2008;15(1):31-8.

[19] Fadlalla I., Abdel A., and Ibrahim M. Antimicrobial susceptibility of Salmonella serotypes isolated from human and animals in Sudan. Journal of Public Health and Epidemiology. 2012;4(1):19-23.

[20] Yang H. CG, Zhu Y., Liu Y., Cheng J., Hu L. Surveillance of Antimicrobial Susceptibility Patterns among Shigella Species Isolated in China during the 7-Year Period of 2005-2011. Ann Lab Med. 2013;33:111-5.

[21] Yismaw G., Kassu A. A five-year antimicrobial resistance pattern observed in Shigella species isolated from stool samples in Gondar University Hospital, northwest Ethiopia. Ethiop J Health Dev. 2006;20(3):194-8.
[22] Kalantar E., Naseri M.H., and Torabi V.Antibiotic resistance patterns of STEC and ETEC strains: A study on frozen foods of animal origin and children with acute diarrhea. $J$ Microbiol Infect Dis. 2013;3(1):31-5.

[23] Olufemi I. O. The incidence and antibiotics susceptibility of Escherichia coli O157:H7 from beef in Ibadan Municipal, Nigeria. African Journal of Biotechnology. 2010;9(8):1196-9.

[24] Nigusse D., Kumie A. Food hygiene practices and prevalence of intestinal parasites among food handlers working in Mekelle university students' cafeteria, Mekelle. Global Advanced Research Journal of Social Science. 2012;1(4):65-71.

[25] Zeru K, Kumie A. Sanitary conditions of food establishments in Mekelle town, North Ethiopia. Ethiop $J$ Health Dev. 2007;21(1):3-11. 